\title{
Alimentación, Salud y Comunicación: hacia una agenda de investigación convergente en Argentina
}

\author{
Flavia Demonte \\ Profesora Adjunta en Facultad de Periodismo y \\ Comunicación Social \\ Universidad Nacional de La Plata (FPyCS, UNLP).
}

\section{Palabras clave}

Resumen

Alimentación

salud

comunicación

investigación

Argentina

\begin{abstract}
El objetivo de este artículo es proponer algunas líneas de investigación sobre alimentación, salud y comunicación, partiendo de la necesidad de la interrelación entre las tres áreas temáticas en un contexto de industrialización alimentaria, medicalización extendida y de perfiles epidemiológicos complejos en Argentina. Teniendo en cuenta ese contexto, se identifican y proponen líneas de investigación que expresan la especificidad de la mirada comunicacional frente a los aportes de otras disciplinas al campo de la alimentación y la salud. Se concluye con los desafíos que implican los temas, problemas y las prácticas investigativas propuestas.
\end{abstract}

Autor para correspondencia: Flavia Demonte. Instituto de Altos Estudios Sociales- Universidad Nacional de general San Martín. (IAES, UNSAM Consejo Nacional de Investigaciones Científicas y Técnicas (CONICET) Universidad Nacional de la Plata (UNLP) Argentina. Correo electrónico: flaviademonte@yahoo.com.ar 


\section{Food, Health, and Communication: towards a research agenda convergent Argentina}

\begin{tabular}{l}
\hline Keywords \\
\hline Food \\
health \\
communication \\
research \\
Argentina
\end{tabular}

\section{Cómo citar el artículo}

Demonte F. (2015). Alimentación, Salud y Comunicación: hacia una agenda de investigación convergente en Argentina. Revista de Comunicación y Salud, Vol. 5, pp. 83-98.

DOI: http://doi.org/10.35669/revistadecomunicacionysalud.2015.5.83-98 


\section{Alimentación y Salud: cuestiones convergentes, discursos contradictorios}

En un contexto en el que la industrialización de la alimentación a partir del siglo XX ha modificado profundamente los modos de producir, distribuir, acceder y consumir los alimentos en Argentina pero también en otros países, este artículo propone algunos posibles alineamientos de investigación sobre alimentación, salud y comunicación, partiendo de la necesidad de la interrelación entre las tres áreas temáticas frente a la persistencia de perfiles nutricionales complejos, particularmente en Argentina -aunque no exclusivamente-.

El aumento del repertorio alimentario a partir de procesos de modernización de la agricultura e industrialización agroalimentaria; los cambios en el procesamiento y en la distribución a partir de nuevas técnicas de conservación, mecanización, transporte, con la consecuente desestacionalización ${ }^{2}$, deslocalización ${ }^{3}$ y homogeneización de las dietas; las modificaciones en la comensalidad ${ }^{4}$ y la existencia de patrones epidemiológicos complejos con problemáticas nutricionales superpuestas son algunas de las tendencias de la alimentación industrializada.

Estas tendencias expresan cambios profundos en el sistema social en su conjunto que están relacionados en un escenario más macro con las transformaciones en el modelo de acumulación capitalista, con la industrialización y el desarrollo de innovaciones tecnológicas, con la urbanización del espacio, con las transformaciones en el mercado laboral y la masiva incorporación de la mujer al trabajo remunerado; con las variaciones de la composición familiar; con los nuevos valores respecto de la salud y el cuerpo y la hegemonía de la delgadez como ideal de belleza, que permitieron el pasaje de la "comida nutritiva" de los años 60 a la comida sana en este fin y principio de siglo, etc.

De acuerdo con Fischler (1995) la modernidad alimentaria aporta un flujo continuo y casi inagotable de comida; pero como hemos visto también expresa un cambio acelerado y una crisis de las costumbres culinarias y de mesa. Estos cambios modificaron -y aun lo hacen- los comportamientos alimentarios de la población (Contreras y Gracia Arnáiz, 2005). La estructuración y composición de las comidas, el tipo de productos consumidos, las maneras de conservarlos y cocinarlos, los lugares donde se come, las normas de mesa o los trabajos y valores asociados a las prácticas alimentarias responden a nuevas lógicas.

Asimismo, estos procesos juegan un rol fundamental en la perturbación de la alimentación y en las causas de las problemáticas nutricionales más prevalentes. Según Fischler (1995) la crisis de la comensalidad tiene al menos un efecto probado: bajo ciertas circunstancias, agrava las patologías que probablemente ha contribuido a determinar. Nos estamos refiriendo, también como tendencia, a la sobrealimentación y a la subalimentación que, en términos generales, caracteriza el consumo alimentario de algunos grupos poblacionales. Aunque de procedencia diversa, y con significaciones muy diferentes, la malnutrición no ha abandonado ni a los países industrializados ni a los más pobres. Si en los primeros la malnutrición tiene su origen en el exceso de consumo de nutrientes, en los segundos lo tiene en el defecto parcial o total de los nutrientes.

La malnutrición característica de los países industrializados se relaciona actualmente con el incremento de enfermedades coronarias, cerebrovasculares y óseas, obesidad, anemia, neoplasias, diabetes, o caries, e incluso con el de otras que presentándose en forma de problemas psicológicos y comportando alteraciones en el comportamiento de las comidas, ocasionan problemas de salud, como es el 
caso de la bulimia y la anorexia nerviosa. Todas ellas constituyen las denominadas enfermedades de la sociedad de la abundancia (Gracia Arnáiz, 2008). En medio de la abundancia contemporánea los problemas de salud han sido desplazados desde aquellos relacionados con la desnutrición a los relacionados con la sobrealimentación.

En Argentina, como expresión de estos procesos, estamos asistiendo a la presencia de perfiles epidemiológicos complejos evidenciados en diferentes estudios llevados a cabo por el Ministerio de Salud de la Nación. La Encuesta Nacional de Nutrición y Salud (ENNyS, 2007) y las sucesivas Encuestas sobre Factores de Riesgo para Enfermedades No Transmisibles (ENFR, 2006, 2011, 2013) evidenciaron la existencia de un patrón nutricional complicado: anemia y deficiencia de hierro, baja talla, y sobrepeso y obesidad constituyen las alteraciones de los estados nutricionales más prevalentes, observadas en todos los grupos etarios relevados pero afectando de manera más extendida a la población de bajos ingresos y de menor nivel educativo.

Como afirma Aguirre (2007) el hambre, a diferencia de otros momentos históricos, no está asociado a 'no comer' sino a 'comer mucho' pero de ciertos alimentos poco densos, nutricionalmente hablando. Por lo tanto, no nos equivocamos si comenzamos a hablar de obesidades ya que, como se polarizó la sociedad, también se polarizaron los cuerpos y sus causas, apareciendo una obesidad de la abundancia y una obesidad de la escasez (Aguirre, 2010).

No obstante ello y de manera superpuesta y contradictoria, se ha desarrollado durante estos últimos años un insistente discurso nutricional sobre la relación entre alimentación y salud. Según Contreras y Gracia Arnáiz (2005) existen estándares de la buena alimentación y se recomienda de manera masiva a través de los medios de comunicación que se mantenga una dieta prudente, equilibrada, racional o saludable que contribuya a preservar la salud. El alimento se ha ido convirtiendo en medicamento y la alimentación ya no responde a la necesidad de satisfacer el hambre o la necesidad de energía sino el hambre de salud. Los transgénicos, la nutrigenómica ${ }^{5}$, la suplementación, la fortificación y los alimentos funcionales ${ }^{6}$ son otro tipo de mercancía alimentaria que genera nuevas construcciones simbólicas y una resignificación de la comida y los alimentos en la cultura alimentaria actual (Aguirre, 2011) tanto en Argentina como en otros países.

Ello expresa una matriz sociocultural -que no es nueva- y que se vincula con procesos de medicalización creciente, proponiendo otras formas de entender el cruce entre este campo y el de la salud. La relación entre la alimentación y la salud de la población no constituye una relación reciente. Remite a la idea de los alimentos o como fuentes de salud o como fuentes de enfermedad, como una dimensión importante para prevenir enfermedades, proteger o restablecer la salud. Remite también a que los alimentos y comportamientos alimentarios sean objeto de incumbencia médica (Gracia Arnáiz, 2007). De acuerdo con Contreras y Gracia Arnáiz (2005), todas las sociedades han desarrollado a lo largo de su historia, creencias y/o conocimientos, más o menos empíricos, relativos al valor de determinados alimentos y a la conveniencia de los mismos según las particularidades de los individuos relativas al sexo, la edad, al organismo, la actividad, a las circunstancias (físicas, psicológicos, sociales y culturales). Asimismo, la larga tradición de la medicina occidental de proveer información y consejos sobre la cantidad y la composición de la comida sana, la regulación del peso y la prevención de enfermedades ha contribuido a privilegiar las funciones 
biológicas de los alimentos a lo largo del tiempo y, dada su influencia en otros sistemas médicos, también del espacio. Que la interpretación biomédica enfatice los aspectos fisiológicos en detrimento de los sociales responde a una lógica que se ha construido al compás de la medicalización de la alimentación (Gracia Arnáiz, 2007).

Este proceso de medicalización en el campo alimentario se encuentra íntimamente ligado a la representación del cuerpo y a los efectos de la incorporación de los alimentos en él. Según Fischler (1996) todo alimento tiene un efecto en el cuerpo, bueno o malo y, en este sentido, el principio de incorporación está estrechamente relacionado con la idea misma de medicina: todo alimento es buena o mala medicina. Según las representaciones decimonónicas, el cuerpo-máquina necesitará un "carburante" -los alimentos- para funcionar, es decir, para producir energía. Esta construcción, la del cuerpo-máquina que funciona gracias a los alimentos traducidos en sus componentes -proteínas, glúcidos y lípidos- ${ }^{8}$, acaba haciendo desaparecer, por lo menos oficialmente, la vieja teoría hipocráticagalénica que concebía al alimento como un todo que pertenece a un lugar, que tiene una historia y que puede, tanto desde el punto de vista preventivo como terapéutico, restablecer el estado de salud a partir de la obtención del equilibrio perdido (Guidonet, 2007).

Nos encontramos con dos procesos paralelos que se complementan, reforzándose. Por una parte, la mirada y el saber médico-nutricional penetran en el cuerpo, transforman su representación, fomentando el control sobre el mismo. Por otro lado, la visualización del interior del alimento y su posterior descomposición se hace efectiva.

No obstante lo antiguo de estos procesos, en la actualidad, con los avances de los conocimientos vinculados con la nutrición y el desarrollo de la biotecnología han cobrado otro impulso ${ }^{7}$. Los alimentos y la alimentación ya no responden a la necesidad de satisfacer el hambre o la necesidad de energía sino el hambre de salud (Contreras y Arnáiz, 2005). El énfasis no sólo está puesto en el aporte de nutrientes suficientes que aseguren la supervivencia de una persona, satisfacer sus necesidades metabólicas y complacer placenteramente su sensación de hambre y bienestar, sino también se acentúa en la potencialidad de los alimentos para la promoción de la salud, mejorar el bienestar y reducir el riesgo de enfermedades (Menéndez Patterson, 2008).

"Producto de ello, nuestras sociedades contemporáneas se interesan por saber qué comemos (...) para, luego, recomendar qué debemos comer" (Contreras y Gracia Arnáiz, 2005: 461). Así, en el terreno de lo alimentario el discurso médico-nutricional impone un orden moral (Guidonet, 2007). Y ese orden moral es el resultado también de una construcción social: lo que es definido como saludable en un momento dado, puede no serlo en otro (Díaz Méndez y Gómez Benito 2008) ${ }^{9}$. Este proceso es retomado por gran cantidad de actores sociales que se erigen también como "expertos". A la oferta en el mercado de alimentos "funcionales" se suman las recomendaciones de nutricionistas y médicos, pero también los medios de comunicación y la propia industria alimentaria participan de este proceso. En tanto no sabemos qué comemos, debe haber una instancia de información que advierta 'qué es esa cosa nueva' que ha salido al mercado. Los medios de comunicación juegan un rol central como espacios en los que se difunde información sobre alimentación y salud y, en ocasiones, como actores que forman parte de los debates actuales (Aguirre, 2011) ${ }^{10}$. 
Esto es coherente con una sociedad en la que la preocupación por la salud asociada a la moderación, la variedad y el equilibrio dietético- se relaciona con la preocupación por la estética y el ideal de la delgadez y la cuestión de la estética se entrecruza y contradice con la sobreabundancia alimentaria, con la idea de comer con placer todo lo que ofrece la industria alimentaria. Así salud, estética y hedonismo (Contreras y Arnáiz, 1995) son los tres mensajes contradictorios que, dependiendo del sector de ingresos al que se pertenezca, se integrarán al discurso sobre la alimentación actual en un contexto de obesidad extendida.

\section{Alimentación, Salud y Comunicación: líneas de investigación propuestas}

En este contexto, en Argentina se ha venido investigando, debatiendo y problematizando desde diferentes ámbitos (político-técnicos, académicos, mediáticos, empresariales y desde organizaciones y movimientos sociales) y con diferentes énfasis, la matriz alimentaria y las principales consecuencias no sólo sanitarias sino también económicas, sociales, políticas, culturales y ambientales que conllevan determinados modos de producir, distribuir, acceder y consumir los alimentos. Por tanto, podemos afirmar que la alimentación en relación con la salud está en la agenda social, mediática y política (Autor, 2015). También se expresa en las preocupaciones de los equipos de salud que cotidianamente se encuentran con diferentes problemáticas nutricionales en el marco de contextos discursivos contradictorios.

Como ha sucedido con otros fenómenos de naturaleza compleja, ya no se discute que la alimentación y su relación con la salud, deban ser estudiadas atendiendo a una perspectiva interdisciplinaria. Teniendo en cuenta ello, la economía, la política, la historia, la medicina y la nutrición, la antropología y la sociología, entre otras disciplinas, han aportado conocimiento de manera agregativa y novedosa. La indagación bibliográfica que hemos llevado nos permite citar algunos ejemplos vinculados específicamente con la alimentación y la salud: algunas investigaciones de autores argentinos provenientes fundamentalmente de la antropología alimentaria y de la salud ${ }^{11}$ han analizado la relación compleja entre la salud y la alimentación, intentando no limitarla al aspecto biológico-nutricional (Hintze, 1991), han investigado sobre los principios de incorporación de los alimentos y su relación con las visiones sobre el cuerpo según sectores de ingresos (Aguirre, 2005), han recopilado información acerca de nuevas ideas sobre la búsqueda de la salud y el bienestar y el desplazamiento, aunque conviviendo de manera contradictoria, de la comida "nutritiva" a la comida "sana" (Álvarez y Pinotti, 2000), han constatado la presencia de "alimentos creados" por parte del sistema científico tecnológico y apropiado por la industria alimentaria (Aguirre, 2011, 2010) por citar sólo algunos ejemplos.

A diferencia de estas otras disciplinas, y si bien el campo de la comunicación en salud es prolífico y prometedor tanto en lo referido a la investigación como al terreno de la experiencia, resulta significativo que encuentre poca o nula presencia en la agenda de la investigación inscripta en el campo de la comunicación, excepto algunas excepciones, dadas por tesis de grado y posgrado, especialmente.

De este modo, para ensayar algunas líneas de investigación, debemos tener en cuenta que cualquier intento de investigar desde una mirada comunicacional la relación entre la alimentación y la salud en un contexto de industrialización 
alimentaria y medicalización extendida como el actual, deberíamos considerar una serie de supuestos como puntos de partida aportados por otras disciplinas.

- La cuestión alimentaria actual tiene poco que ver con la escasez y mucho con la abundancia alimentaria expresada en un aumento en la disponibilidad de alimentos para ser potencialmente consumidos.

- La industria alimentaria produce diferentes tipos de productos según el sector de ingresos. Así, sigue a la nueva cocina -ligera, frugal, desgrasada-, a la medicina y la nutrición -con sus recomendaciones que, casualmente, se corresponden con las preferencias y los comportamientos de los grupos de ingresos altos-, pero también vende a los sectores de bajos ingresos productos baratos con exceso de grasas e hidratos, lo que explica la obesidad en contextos de pobreza (Aguirre, 2005). Junto con los avances científico-tecnológicos, recoge todos estos discursos para vender sus productos, generando mensajes y difundiéndolos a través de los medios de comunicación.

- Las organizaciones científicas renuevan sus recomendaciones y las hacen circular por diversos medios.

- Los medios de comunicación en sus diversas variantes, formatos y géneros son dominios en los que todos estos discursos se difunden y promueven, recreando necesidades para las que la industria alimentaria, coadyuvada por el sistema científico-tecnológico, sigue ofreciendo nuevos alimentos.

- Los conocimientos acerca de la alimentación y la nutrición, los desarrollos tecnológicos y las aplicaciones biotecnológicas se han profundizado a la vez que democratizado. Cada vez más la población tiene mayor información sobre la alimentación saludable -aunque siente temor frente al desconocimiento de los métodos de producción- pero persisten prácticas alimentarias poco saludables.

- La persistencia de perfiles nutricionales complejos y contradictorios está relacionada con causas complejas y multicausales -condicionantes económicos, culturales y políticos afectan todo y a todos: el acceso a los recursos, las relaciones de género, las formas de entender la enfermedad o los cuidados del cuerpo-, aquejando a poblaciones con niveles de ingresos diferenciados.

- El Estado es un actor con capacidades tecno-políticas para regular la matriz alimentaria y evitar las consecuencias en la salud de la población, pero pocas veces hace uso de ellas frente a actores poderosos, limitando sus intervenciones a producción de información sobre los perfiles nutricionales, programas de alimentación saludable y campañas de información y comunicación con escasos resultados.

- Los equipos de salud se enfrentan a nuevos escenarios sociales, culturales, sanitarios con capacidades limitadas para incidir en los saberes y en las prácticas de los individuos y las poblaciones.

Así, discurso hedonista, discurso estético pero sobre todo el discurso médico confluyen contradictoriamente, limitando, permitiendo e incluso promoviendo su consumo de ciertos alimentos (Contreras y Gracia Arnáiz, 2005). 
Frente a este escenario, y circunscribiéndonos al campo de la Comunicación y Salud (CyS), y a todas sus líneas y ámbitos de trabajo, cabría preguntarnos en primer lugar ¿qué especificidad tendría una investigación sobre Alimentación y Salud desde el punto de vista comunicacional que la diferencie de otros aportes disciplinares?

Cuando estudiamos la dimensión comunicacional de las cuestiones vinculadas con la alimentación en relación con la salud, no estudiamos tanto los aspectos objetivos de estas cuestiones sino las construcciones simbólicas -la producción, circulación y recepción de sentidos sociales- que individuos, familias, comunidades, poblaciones o equipos de trabajo ponen en juego en estos temas y que operan como guías que estructuran las diversas prácticas vinculadas con la salud y la alimentación. Ello por supuesto nos acerca a disciplinas como la sociología y la antropología.

Teniendo en cuenta una posible especificidad, en segundo lugar, cabría preguntarnos ¿cuáles serían esos necesarios lineamientos de investigación? Sin el propósito de que sea exhaustivo ni tampoco novedoso, se propone un listado de posibles temas y cuestiones susceptibles de ser indagados desde una perspectiva comunicacional, asumiendo la relatividad de los diferentes contextos sociales, económicos, políticos, culturales, sanitarios, discursivos e investigativos:

Industria Alimentaria. Comercialización, Publicidad y Discurso Publicitario

- Análisis de las técnicas de distribución y comercialización de productos alimentarios vinculados con la búsqueda de salud y bienestar, por ejemplo, alimentos funcionales.

- Análisis de la publicidad alimentaria según dimensiones y categorías específicas, destinada a público tanto adulto como infantil y su relación con la promoción de prácticas alimentarias saludables.

Medios de Comunicación. Discurso Mediático

- Análisis de los mensajes y discursos de los medios de comunicación en todas sus variantes, formatos y géneros, las representaciones que recrean y reproducen sobre los diferentes temas vinculados con la alimentación en su relación con la salud. Análisis de la construcción del discurso público sobre alimentación y salud y análisis de la agenda mediática.

- Estudios sobre la revalorización de figuras -como los cocineros y cocineras como personajes mediáticos- y su incidencia en la promoción de prácticas alimentarias saludables.

Organizaciones no gubernamentales. Debates y propuestas de acción sobre seguridad y soberanía alimentaria relacionadas con la cuestión sanitaria.

- Análisis comunicacional de los debates, las propuestas de acción y de las recomendaciones de los organismos técnicos internacionales vinculados con el campo temático (Organización Mundial de la Salud, Organización Panamericana de la Salud, Organización de las Naciones Unidas para la Alimentación y la Agricultura, etc.). 
- Análisis comunicacional de los debates, las propuestas de acción y de las recomendaciones de organizaciones y movimientos sociales y comunitarios (internacionales y nacionales).

Escuela y Academia. Debates, enfoques. Propuestas formativas e investigativas.

- Análisis comunicacional de las propuestas pedagógicas -propuestas curriculares, estrategias didácticas, etc.- vinculadas con la alimentación y la salud destinadas a la población infantil frente a los perfiles nutricionales de la población infantil (prevalencia de sobrepeso y obesidad).

- Análisis del campo académico en relación con las propuestas formativas en sus diferentes variantes y niveles de grado y posgrado, capacitación en servicio vinculadas con la alimentación y la salud en carreras afines (medicina, enfermería, nutrición, psicología, antropología, sociología, comunicación, por citar sólo algunas).

- Relevamiento de las líneas de investigación, la producción científico-tecnológica (mesas y ponencias en eventos científicos, tesis, libros, artículos en revistas científicas y de divulgación), y su relación con las políticas públicas.

Estado. Políticas Públicas, Servicios y Equipos de Salud, Alimentación, Salud y Comunicación. Discurso público-político y discurso sanitario o biomédico.

- Análisis de procesos de movilización e incidencia en políticas públicas de diferentes actores sociales para la sanción de leyes, para la demanda de acciones programáticas o para la organización de servicios y acciones vinculados con la prevención, promoción, asistencia y/o rehabilitación de padecimientos vinculados con la alimentación.

- Análisis de los lineamientos comunicacionales de los marcos legales, políticas, programas, proyectos o protocolos de atención vinculados con la alimentación y la salud así como de campañas, materiales de sensibilización, información y comunicación en diferentes ámbitos, niveles y jurisdicciones. Validación de materiales de información y comunicación.

- Análisis de gestión de estrategias y procesos comunicacionales vinculados con las políticas alimentarias y las políticas sanitarias en diferentes ámbitos, niveles y jurisdicciones.

- Estudios sobre las barreras culturales que obstaculizan que la población acceda a servicios y prestaciones alimentarias y sanitarias. Evaluaciones de servicios y prestaciones en su dimensión informacional y comunicacional.

- Análisis de la información, los discursos y las prácticas de los equipos de salud en relación con la alimentación y la salud.

Individuos, familias, comunidades y poblaciones. 
- Análisis de la información -que necesitan y/o que disponen-, los discursos y las prácticas de la población diferenciada por ciclos vitales, por género, niveles de ingresos, por padecimientos, por tipos y prácticas de alimentación -vegetarianismo, macrobiótica, veganismo, naturismo, ayuno, etc. en relación con la alimentación y la salud y la incidencia de ellos en los perfiles epidemiológicos.

- Identificación y análisis de audiencias y consumos culturales para planificar intervenciones comunicacionales en comunicación y prácticas alimentarias saludables.

Cabe aclarar que estas líneas de investigación están pensadas fundamentalmente desde los actores intervinientes. Si se especifica por temas y subtemas, en relación con la alimentación, la salud y la comunicación, es posible proponer los siguientes nuevamente, no es exhaustiva-, siempre retomando la dimensión comunicacional de los mismos:

- Aquellos vinculados con los consumos alimentarios -las prácticas y sus simbolizaciones- en términos de factores de riesgo (tipos de alimentos y estilos y modos de vida) para el desarrollo de otras enfermedades.

- Aquellos vinculados con problemáticas y grupos específicos (Desnutrición en todas sus variantes, Obesidad, Anemia, Síndrome Urémico Hemolítico, Celiaquía, Trastornos de la Alimentación como bulimia y anorexia) y sus simbolizaciones.

- Aquellos vinculados con nuevos avances científicos, aplicaciones biotecnológicas y su relación con los diferentes actores sociales y con la percepción social respecto de los mismos y de los riesgos respecto de sus consumos.

- Aquellos vinculados con la matriz agroalimentaria (producción, distribución, acceso), la seguridad y la soberanía alimentaria y sus implicancias socio-sanitarias como temas debatibles en el espacio público, político y mediático.

- Aquellos vinculados con las políticas, programas, servicios y prestaciones del Estado y de los equipos de salud.

Más allá de que estudiemos estos u otros temas/cuestiones/problemas, lo cierto es que debemos partir de reconocer la compleja coyuntura socioeconómica, la matriz sociocultural, saber cómo están incidiendo en las representaciones y prácticas alimentarias en el surgimiento y/o persistencia de ciertos problemas alimentarionutricionales.

Del análisis de la complejidad del contexto de industrialización alimentaria y medicalización creciente descripto en apartados precedentes y del reconocimiento que realizan Contreras y Gracia Arnáiz (2005) en relación con la proliferación de medios y sistemas difusores de modelos alimentarios y el consecuente eclecticismo discursivo sobre alimentación y salud pasamos a la construcción de posibles temas de investigación para armar una agenda en la que converjan estos temas y cuestiones mirados desde una perspectiva comunicacional. El eje específico puede ser considerado a partir de aspectos diferentes pero complementarios que deben leerse desde diferentes niveles y actores: la producción, circulación, recepción de sentidos sociales vinculados con la salud y la alimentación. La población en general recibe, recrea, resignifica, se apropia de -también produce- discursos de diverso orden sobre cocina, estética, dietética y salud a través de diversos medios: familia, administración pública, asociaciones de consumidores, académicos y técnicos, 
industria alimentaria, publicidad, medios de comunicación, etc. Esta situación, según Contreras y Gracia Arnáiz (2005), contribuye a que las elecciones y preferencias alimentarias se vean alentadas o condicionadas por una multiplicidad de mensajes sobre los alimentos, sus técnicas de producción y las consecuencias que conlleva en la salud consumirlos o no consumirlos, sobre las situaciones de consumo. Los discursos médicos se mezclan, se enfrentan o confunden con los discursos gastronómicos, los regímenes de adelgazamiento se juntan con los de recetas y los manuales de nutrición y salud con las guías gastronómicas. Analizar ese peso del contexto discursivo en las prácticas sociales es lo que nos convoca a los comunicadores a investigar sobre estos temas.

\section{Desafíos de líneas de investigación convergentes}

Para trabajar en líneas de investigación que se integren a una agenda de investigación en comunicación tan promisoria como fructífera, deberíamos trascender algunas modalidades de investigar que podríamos denominar como clásicas. Algunos de esas cuestiones están relacionadas con los temas objeto de estudio, con su abordaje -estrategias metodológicas- y con las formas de trasferir y compartir esos conocimientos en diferentes ámbitos.

- Del análisis de representaciones y prácticas asociadas a problemáticas específicas al análisis de las problemáticas en relación, teniendo en cuenta la complejidad de los patrones alimentarios antes aludida, las condiciones y los modos de vida.

- Del análisis de representaciones -a través de análisis de discurso periodístico, publicitario, etc.- en la prensa gráfica al análisis de representaciones recreadas en la comunicación multimedia (prensa gráfica, televisión, redes sociales, etc.).

- Del análisis de miradas circunscriptas a un grupo poblacional o a actores por separado a análisis relacionales (población y equipos de salud en contextos interactivos e interaccionales; confluencia entre industria alimentaria e industria publicitaria; confluencia entre recomendaciones biomédicas e industria alimentaria, etc.), para comprender la lógica de los procesos de medicalización de las prácticas alimentarias.

- De la investigación académica a la investigación que se articule con la formulación, implementación y evaluación de políticas alimentarias y sanitarias.

Por lo tanto, para estar a la altura de expectativas no sólo académicas sino también sociales, políticas y técnicas, es necesario identificar los desafíos que implican los pasajes enunciados precedentemente para adecuar las agendas de investigación a problemas y cuestiones relevantes socialmente.

El primer desafío está relacionado con el reconocimiento y el abordaje de un objeto que necesariamente debe construirse interdisciplinariamente y que reaparece en diversos formatos, géneros y tipos de discursos. Gracia Arnáiz (2008) reconoce la dificultad de remontar tradiciones disciplinares, ciertas formas de ver las cosas, ciertas metodologías de investigación. Pero es necesario incorporar otras miradas que sobre el mismo tema están ofreciendo otras disciplinas, integrándolas a la construcción y al abordaje de un objeto interdisciplinario. Los profesionales de la comunicación que se dediquen a investigar los temas aquí tratados deberán adquirir 
conocimientos vinculados con el campo biomédico, psicológico, sociológico y antropológico, en tanto que éstos, integrados a la formación en comunicación y en investigación, permitirán abordar los objetos de investigación construidos así como brindar recomendaciones técnicas para la formulación e implementación de políticas públicas.

Un segundo desafío importante, relacionado con el anterior, es la necesidad de aprender a identificar problemáticas, prácticas, procesos, productos vinculados con la alimentación y la salud que son susceptibles de una mirada y un análisis comunicacional y abogar por la importancia que adquiere realizar investigación vinculada con esta dimensión. Esto implica construir nuestro objeto de análisis en el marco de un proceso y una problemática más amplia; a la vez que bregar por la importancia e implicancia que asume en sociedades complejas como las nuestras. No resulta fácil esta tarea en un contexto complejo que, como hemos dicho, requiere para su análisis e intervención de la articulación de diversas disciplinas dentro de un marco integrado (Bruno y Autor, 2013). Ello permitirá identificar también los aportes de la comunicación a la construcción de ese objeto interdisciplinario.

El tercer desafío está relacionado con la necesidad de realizar mapeos conceptuales y metodológicos para identificar nuevas modalidades de lectura y abordaje de ese objeto construido interdisciplinariamente. Ello implica apelar no sólo a paradigmas, perspectivas, enfoques que nos permitan agudizar la mirada sobre procesos complejos, sino especialmente apelar a estrategias metodológicas nos permitan abordar rigurosamente esos objetos y procesos.

Un último desafío está relacionado con la vinculación entre investigación y el proceso de transferencia de resultados para la toma de decisiones. Sin desconocer la riqueza programática de las intervenciones comunicacionales en alimentación y salud, ello no ha tenido, como lo hemos dicho en párrafos más arriba, un desarrollo similar en términos de investigación en el marco de las políticas alimentarias y sanitarias. Ello implica entonces un doble reto: abogar por la necesidad de investigar para la acción y/o la toma de decisiones (Bruno y Autor, 2013) y disputar espacios en relación con los aportes singulares y específicos que desde la comunicación se pueden realizar en el cruce de dos campos que necesariamente convergen, como el de la alimentación y la salud.

\section{Conclusiones}

El objetivo de este artículo consistía en proponer algunas líneas de investigación sobre alimentación, salud y comunicación, partiendo de la necesidad interrelacionar las tres áreas temáticas en un contexto de industrialización alimentaria, medicalización extendida y de perfiles epidemiológicos complejos en Argentina.

Del análisis de la complejidad del contexto en el que nos encontramos pasamos, de acuerdo con la indagación y análisis bibliográfico realizado, al reconocimiento de la vacancia de líneas de investigación vinculadas con estos temas, al menos en Argentina. Este reconocimiento motivó a proponer posibles temas de investigación 
para armar una agenda en la que converjan estos temas y problemas mirados desde una perspectiva comunicacional y que puedan dar lugar a investigaciones necesarias no sólo desde el punto de vista académico sino especialmente desde el punto de vista sanitario, social, político y económico.

En este sentido no sólo se mencionaron los temas más destacados, intentando identificar la especificidad comunicacional respecto de otras disciplinas, sino también intentando identificar los desafíos -epistemológicos, metodológicos, políticos- implicados en la construcción de esa agenda y en su adecuación a problemas social y sanitariamente relevantes. Por tanto y para concluir lo más significativo es el desafío político vinculado con incidir en diversos espacios -no sólo académicos- para abogar por la importancia y los aportes que puede hacer la comunicación a los campos de la alimentación y la salud. Esta estrategia -de incidencia y abogacía- sólo puede configurarse desarrollando investigaciones inscriptas en los diversos ámbitos en los que se desempeñan los comunicadoresque expresen nuevos intereses profesionales, nuevas maneras de entender la producción de conocimientos y las prácticas investigativas -pertinentes, relevantes, interdisciplinarias, con cierta profundidad analítica, etc.-. Pero quizá lo más importante es que deben dejar claro que la indagación de la dimensión simbólica de las prácticas sociales constituye un aspecto sustancial en nuestras sociedades que nos permite analizar los discursos y las prácticas vinculadas con la alimentación y la salud y comprender y explicar cómo inciden en la existencia de perfiles epidemiológicos en un contexto de industrialización alimentaria y de medicalización extendida en Argentina.

\section{NOTAS}

${ }^{1}$ El presente artículo se inscribe en el proyecto "Comer para vivir (¿mejor?). Análisis de las representaciones sociales sobre la alimentación y su vinculación con la salud/enfermedad en la Argentina reciente (2009-2014)". Se financia a través de una beca postdoctoral otorgada por el Consejo Nacional de Investigaciones Científicas y Técnicas (CONICET), y se desarrolla en el Instituto de Altos Estudios en Ciencias Sociales de la Universidad Nacional de General San Martín (IDAES/UNSAM), Argentina, bajo la dirección de la Dra. Patricia Aguirre. El proyecto se inició en el mes de abril de 2015 y se encuentra en curso. Asimismo, el artículo retoma algunas conclusiones de la Tesis de Maestría en Políticas Sociales y de la Tesis de Doctorado en Ciencias Sociales.

2 El término "desestacionalización" hace referencia a que las nuevas técnicas de conservación permiten superar las fluctuaciones estacionales que han acompañado desde siempre a la alimentación humana (Aguirre, 2011) y que los alimentos estén disponibles, independientemente de la estación del año.

${ }^{3}$ El término "deslocalización" de las dietas hace referencia a las consecuencias de las formas y técnicas de producción actual en el consumo. En otras palabras, al tiempo que se multiplicó y racionalizó la producción, se diversificaron las posibilidades de consumo, trayendo como consecuencia que los alimentos perdieran su anclaje con el medio ambiente circundante (Aguirre, 2011). 
${ }^{4}$ El término "comensalidad" hace referencia a las reglas vinculadas con las maneras de compartir las comidas: contempla la organización y jerarquía de las edades y los géneros, la separación de platos de consumo diario y de prestigio, la cantidad de comidas diarias, sus horarios, la cantidad y formato de los platos, entre otras cuestiones (Aguirre, 2011 citado de Fernández-Armesto, 2001).

5 Es el estudio de la relación entre el estímulo nutricional y la respuesta de los genes. Los estudios en este campo pueden llevarnos en el futuro a ingerir alimentos de acuerdo con nuestras diferencias genéticas y a la elaboración de dietas personalizadas mediante las cuales se consiga una alimentación más saludable que revierta en cambios fenotípicos favorables, con la consiguiente reducción de la incidencia de mortalidad por las distintas enfermedades (Menéndez Patterson, 2008).

6 Se denominan "alimentos funcionales" a cualquier alimento o ingrediente potencialmente saludable que pueda proveer beneficios a la salud más allá de los nutrientes tradicionales que contiene.

7 Nos referimos a las aplicaciones biotecnológicas. La biotecnología tiene como objeto el estudio científico de los métodos y aplicaciones cuyo soporte son los seres u organismos vivos para la obtención y mejora de los productos tales como alimentos o medicamentos. En el ámbito alimentario dichas aplicaciones afectan principalmente a los vegetales, animales y productos fermentados, aunque el campo de las aplicaciones agrícolas es el que ha experimentado un mayor crecimiento.

$8 \mathrm{Ya}$ en el siglo XX se puso de manifiesto la presencia, las funciones y las necesidades de los minerales y el descubrimiento de los nutrientes esenciales: vitaminas, aminoácidos, minerales, ácidos grasos. A finales del siglo XX comienza a cobrar interés los compuestos bioactivos que no entran en ninguna de las categorías de nutrientes conocidas (esenciales y energéticas). Se definen como compuestos bioactivos a aquellos constituyentes que no son nutrientes y que típicamente se encuentran de forma natural en pequeñas cantidades de vegetales y en alimentos ricos en lípidos (Menéndez Patterson, 2008).

9 Estos investigadores destacan, en el contexto español, la influencia que han tenido la situación del sector agrario, el proceso de industrialización, la industria alimentaria y los objetivos de las políticas agrarias en las recomendaciones alimentarias presentes en el discurso médico-nutricional, dando cuenta de su gran mutabilidad. Al respecto también Contreras y Arnáiz (2008) afirman que las normas dietéticas fluctúan a lo largo del tiempo, según los descubrimientos y el aumento de la influencia social de sus prescriptores.

10 Al respecto, Menéndez Patterson (2008) destaca el papel de los medios de comunicación en la difusión, por ejemplo, de los alimentos funcionales. Afirma que el mundo de la salud y específicamente el de la alimentación han cobrado una especial relevancia en los medios de comunicación que, en muchos casos, se han convertido en una referencia para lectores y asociaciones de pacientes. 
11 Cabe señalar que investigares españoles y franceses han realizado innumerables trabajos y que, seleccionados, han sido oportunamente citados en el presente artículo.

\section{Referencias}

Aguirre, Patricia (2011). La construcción social del gusto en el comensal moderno. En Aguirre, Patricia, Katz, Mónica y Bruera, Matías Comer. Puentes entre la alimentación y la cultura. Buenos Aires: Libros del Zorzal.

Aguirre, Patricia (2010). La comida en los tiempos del ajuste. En Torrado, Susana (dir.) El costo social del ajuste (Argentina 1976-2002). Tomo II. Buenos Aires: Edhasa.

Aguirre, Patricia (2007). Comida, cocina y consecuencias: la alimentación en Buenos Aires. En Torrado, Susana (comp.) Población y Bienestar en la Argentina del primero al segundo Centenario. Una historia del siglo $X X$. Buenos Aires: Edhasa.

Aguirre, Patricia (2005). Estrategias de consumo: qué comen los argentinos que comen. Buenos Aires: Miño y Dávila.

Álvarez, Marcelo y Pinotti, Luisa (2000). A la mesa. Ritos y retos de la alimentación argentina. Buenos Aires: Grijalbo.

Contreras, José y Gracia Arnáiz, Mabel (2005). Alimentación y cultura. Barcelona: Ariel.

Fischler, Claude (2014) [ 1996 ] . Las funciones de lo culinario. En Piaggio, Laura y Solans, Andrea (comps) Enfoques Socioculturales de la Alimentación. Buenos Aires: Akadia.

Fischler, Claude (1995). Gastro-nomía y Gastro-anomia. Sabiduría del cuerpo y crisis biocultural de la alimentación moderna. Gazeta de Antropología Vol 1. ํㅡ 26. pp. 357-379.

Guidonet, Alicia (2007). La antropología de la alimentación. Barcelona: UOC.

Gracia Arnáiz, Mabel (coord.) (2008). Somos lo que comemos. Estudios de alimentación y cultura en España. Barcelona: Ariel.

Gracia Arnáiz Mabel (2007). Comer bien, comer mal: la medicalización del comportamiento alimentario. Salud Pública no 49. pp. 236-242.

Hintze, Susana (1991). Las relaciones entre salud y alimentación: acerca de la autonomía de lo alimentario. Cuadernos Médico Sociales ํำ58, pp. 47-53.

Menéndez Patterson, María Ángeles (2008). Avances científicos en nutrición y alimentación. En Díaz Méndez, Cecilia y Gómez Benito, Cristobal (coord.) (2008) Alimentación, consumo y salud. Col. Estudios Sociales n² 24. Fundación La Caixa. 
Ministerio de Salud de la Nación (2007). Encuesta Nacional de Nutrición y Salud. Documento de Resultados. Buenos Aires: Ministerio de Salud de la Nación.

Ministerio de Salud de la Nación (2006). Primera Encuesta Nacional de Factores de Riesgo para Enfermedades No Transmisibles. Buenos Aires: Ministerio de Salud de la Nación.

Ministerio de Salud de la Nación (2011). Segunda Encuesta Nacional de Factores de Riesgo para Enfermedades No Transmisibles. Buenos Aires: Ministerio de Salud de la Nación.

Ministerio de Salud de la Nación (2013). Tercera Encuesta Nacional de Factores de Riesgo para Enfermedades No Transmisibles. Buenos Aires: Ministerio de Salud de la Nación. 